\title{
An Efficient Algorithm for Solving Hyperbolic Partial Differential Equations with a Nonlocal Conservation Condition
}

\author{
H. Bin Jebreen \\ Department of Mathematics, College of Science, King Saud University, P.O. Box 2455, Riyadh 11451, Saudi Arabia \\ Correspondence should be addressed to H. Bin Jebreen; hjebreen@ksu.edu.sa
}

Received 15 November 2020; Revised 23 December 2020; Accepted 22 February 2021; Published 5 March 2021

Academic Editor: Ahmed Abouelregal

Copyright (c) $2021 \mathrm{H}$. Bin Jebreen. This is an open access article distributed under the Creative Commons Attribution License, which permits unrestricted use, distribution, and reproduction in any medium, provided the original work is properly cited.

In this paper, a numerical scheme based on the Galerkin method is extended for solving one-dimensional hyperbolic partial differential equations with a nonlocal conservation condition. To achieve this goal, we apply the interpolating scaling functions. The most important advantages of these bases are orthonormality, interpolation, and having flexible vanishing moments. In other words, to increase the accuracy of the approximation, we can individually or simultaneously increase both the degree of polynomials (multiplicity $r$ ) and the level of refinement (refinement level $J$ ). The convergence analysis is investigated, and numerical examples guarantee it. To show the ability of the proposed method, we compare it with existing methods, and it can be confirmed that our results are better than them.

\section{Introduction}

The present paper is organized to find the approximate solution of the one-dimensional hyperbolic partial differential equations (HPDEs) with a nonlocal conservation condition [1].

$$
w_{t t}(x, t)-w_{x x}(x, t)=p(x, t), \quad x \in[0,1], 0 \leq t \leq T,
$$

with initial conditions

$$
\begin{aligned}
w(x, 0) & =q(x), \quad x \in[0,1], \\
w_{t}(x, 0) & =g(x), \quad x \in[0,1],
\end{aligned}
$$

Dirichlet boundary condition

$$
w(0, t)=h(t), \quad 0 \leq t \leq T,
$$

and also the integral condition

$$
\int_{0}^{1} w(z, t) \mathrm{d} x=k(t), \quad 0 \leq t \leq T .
$$

Here, $q, p, g, h$, and $k$ are known functions. Without loss of generality, assume that $T=1$.
Mathematical modeling of many physical phenomena leads to HPDEs with boundary and initial conditions and also integral condition replacing the standard boundary condition [2]. This problem appears when studying the dynamics of groundwater, plasma physics, thermoelasticity, and heat conduction [3-5]. The existence and uniqueness of a generalized solution of such an equation were investigated by Mesloub and Bouziani [6]. In this paper, all the known functions of equation (1) and its conditions are assumed to be such that the problem has a unique solution. Numerous studies proposed a variety of numerical and analytical solutions to such equations. Dehghan and Lakestani [1] introduced a numerical scheme based on the cubic B-spline scaling functions to solve (1). In [1], the operational matrices of the derivative and integral were used to reduce the problem to a system of algebraic equations. Several finite difference procedures based on the three-level implicit and explicit schemes are proposed for solving the problem in [5]. In [3], Ang proposed an integrodifferential formulation to find the approximate solution of (1). Ashyralyev and Aggez [7] utilized the stable difference schemes to solve multidimensional hyperbolic equations with nonlocal boundary value. The collocation method based on Jacobi polynomials and Gauss-Lobatto quadrature integration was used in [8]. 
Doha et al. applied shifted Jacobi spectral-Galerkin method to solve the problem [9]. An efficient computational method is proposed for solving hyperbolic partial differential equations based on Chebyshev and Legendre wavelets [10]. In [11], Turkyilmazoglu solved parabolic partial differential equations with nonlocal initial and boundary conditions using a fast and accurate analytic method based on double power series.

Wavelets and their applications are a very powerful tool for solving a variety of equations such as ordinary and partial differential equations and are especially one of the best methods to solve the integral equations with the ability of them at reducing the problem to a sparse linear system. Meanwhile, the wavelet Galerkin method is used widely for solving equations $[2,12-15]$. In this paper, we used interpolating scaling function constructed in [16] following [17], and these bases have been used to solved equations such as ordinary and partial differential equations and also integral equations [18-20]. These bases allow us to have high vanishing moments, compact support, and properties such as orthogonality and interpolation [16].

This paper is organized as follows. Section 2 is devoted to the brief introduction to the interpolating scaling function. In Section 3, Galerkin method based on interpolating scaling functions is used to solve the desired equation, and also, the convergence analysis is investigated. Section 4 is devoted to some numerical examples to show the ability and accuracy of the method.

\section{Interpolating Scaling Functions}

We introduce the subspace $V_{J}^{r}$ as a space of piecewise polynomial bases of degree less than multiplicity parameter $r \geq 0$ that is spanned by

$$
\begin{gathered}
V_{J}^{r}:=\operatorname{Span}\left\{\phi_{j, b}^{k}:=\phi^{k}\left(2^{j} x-k\right), b \in \mathscr{B}_{j}, k \in \mathscr{R}\right\} \subset L^{2}(\Omega), \\
r \geq 0, \quad j \in \mathbb{Z}^{+} \cup\{0\},
\end{gathered}
$$

where two sets $\mathscr{B}$ and $\mathscr{R}$ are defined by

$$
\begin{aligned}
& \mathscr{B}:=\left\{0, \ldots, 2^{J}-1\right\}, \\
& \mathscr{R}:=\{0,1, \ldots, r-1\},
\end{aligned}
$$

and $\left\{\phi^{k}\right\}_{k \in \mathscr{R}}$ are the primal interpolating scaling bases introduced by Alpert et al. [17]. Given nodes $\left\{\tau_{k}\right\}_{k \in \mathscr{R}}$, which are the roots of the Legendre polynomial of degree $r$, the interpolating scaling bases are defined as

$$
\phi^{k}(x)= \begin{cases}\sqrt{\frac{2}{\omega_{k}}} L_{k}(2 x-1), & x \in[0,1], \\ 0, & \text { o.w, }\end{cases}
$$

where $\left\{L_{k}(t)\right\}_{k \in \mathscr{R}}$ are the Lagrange interpolating polynomials at the point $\left\{\tau_{k}\right\}_{k \in \mathscr{R}}$ and $\left\{\omega_{k}\right\}_{k \in \mathscr{R}}$ are the Gauss-Legendre quadrature weights [2, 17]. These bases form orthonormal bases on $\Omega$ with respect to the $L^{2}$-inner product.
Let $J \in \mathbb{Z}^{+} \cup\{0\}$. We consider the uniform finite discretizations $\Omega:=[0,1]=\cup_{b \in \mathscr{B}} X_{J, b}$, where the subintervals $X_{J, b}:=\left[x_{b}, x_{b+1}\right]$ are determined by the point $x_{b}:=b /\left(2^{J}\right)$. Every function $p \in L^{2}(\Omega)$ can be represented in the form

$$
p \approx \mathscr{P}_{J}^{r}(p)=\sum_{b \in \mathscr{B}_{J}} \sum_{k \in \mathscr{R}} p_{J, b}^{k} \phi_{J, b}^{k},
$$

where $\mathscr{P}_{J}^{r}$ is the orthogonal projection that maps $L^{2}(\Omega)$ onto the subspace $V_{J}^{r}$. To find the coefficients $p_{J, b}^{k}$ that are determined by $\left\langle p, \phi_{J, b}^{k}\right\rangle=\int_{X_{I, b}} f(x) \phi_{J, b}^{k}(x) \mathrm{d} x$, we shall compute the integrals. We apply the $r$-point Gauss-Legendre quadrature by a suitable choice of the weights $\omega_{k}$ and nodes $\tau_{k}$ for $k \in \mathscr{R}$ to avoid these integrals $[13,17]$ via

$$
p_{J, b}^{k} \approx 2^{-J / 2} \sqrt{\frac{\omega_{k}}{2}} p\left(2^{-J}\left(\frac{\tau_{k}+1}{2}+b\right)\right), \quad b \in \mathscr{B}_{J}, k \in \mathscr{R} .
$$

Convergence analysis of the projection $\mathscr{P}_{J}^{r}(p)$ is investigated for the $r$-times continuously differentiable function $p \in \mathbb{C}^{r}(\Omega)$ :

$$
\left\|\mathscr{P}_{J}^{r}(p)-p\right\| \leq 2^{-J r} \frac{2}{4^{r} r !} \sup _{x \in[0,1]}\left|p^{(r)}(x)\right| .
$$

For the full proof of this approximation and further details, we refer the readers to [16]. Thus, we can conclude that $\mathscr{P}_{J}^{r}(p)$ converges to $p$ with the rate of convergence $\mathrm{O}\left(2^{-J r}\right)$.

Assume that the vector function $\Phi_{J}^{r}:=\left[\Phi_{r, J, 0}, \ldots\right.$, $\left.\Phi_{r, J, 2^{J}-1}\right]^{T}$ with $\Phi_{r, J, b}:=\left[\phi_{J, b}^{0}, \ldots, \phi_{J, b}^{r-1}\right]$ includes the scaling functions and is called the multiscaling function. Approximation (8) may be rewritten using the vector $P$ that includes entries $P_{b r+k+1}:=p_{J, b}^{k}$ as follows:

$$
\mathscr{P}_{J}^{r}(p)=P^{T} \Phi_{J}^{r}
$$

where $P$ is an $N$-dimensional vector $\left(N:=r 2^{J}\right)$. The building blocks of these bases' construction can be applied to approximate a higher-dimensional function. To this end, one can introduce the two-dimensional subspace $V_{J}^{r, 2}:=V_{J}^{r} \times$ $V_{J}^{r} \subset L^{2}(\Omega \times \Omega)$ that is spanned by

$$
\left\{\phi_{J, b}^{k} \phi_{J, b^{\prime}}^{k^{\prime}}: b, b^{\prime} \in \mathscr{B}_{J}, k, k^{\prime} \in \mathscr{R}\right\} \text {. }
$$

Thus, by this assumption, to derive an approximation of the function $p \in L^{2}(\Omega \times \Omega)$ by the projection operator $\mathscr{P}_{J}^{r}$, we get

$$
p(s, t) \approx \mathscr{P}_{J}^{r}(p)(s, t)=\Phi_{J}^{r T}(s) P \Phi_{J}^{r}(t),
$$

where components of the square matrix $P$ of order $N$ are obtained by

$$
P_{r b+(k+1), r b^{\prime}+\left(k^{\prime}+1\right)} \approx 2^{-J} \sqrt{\frac{\omega_{k^{\prime}}}{2}} \sqrt{\frac{\omega_{k}}{2}} p\left(2^{-J}\left(\widehat{\tau}_{k}+b\right), 2^{-J}\left(\widehat{\tau}_{k^{\prime}}+b^{\prime}\right)\right),
$$


where $\widehat{\tau}_{k}=\left(\tau_{k}+1\right) / 2$. Consider the $2 r$-th partial derivatives of $p: \Omega \times \Omega \longrightarrow \mathbb{R}$ are continuous. Utilizing this assumption, the error of this approximation can be bounded as follows:

$$
\left\|\mathscr{P}_{J}^{r} p-p\right\| \leq \mathscr{M}_{\max } \frac{2^{1-r J}}{4^{r} r !}\left(2+\frac{2^{1-J r}}{4^{r} r !}\right),
$$

where $\mathscr{M}_{\text {max }}$ is a constant [13].

$$
\mathscr{M}_{\max }=\max \left\{\sup _{\xi \in[0,1)}\left|\frac{\partial^{r}}{\partial x^{r}} p(\xi, y)\right|, \sup _{\eta \in[0,1)}\left|\frac{\partial^{r}}{\partial y^{r}} p(x, \eta)\right|, \sup _{\xi^{\prime}, \eta^{\prime} \in[0,1)}\left|\frac{\partial^{2 r}}{\partial x^{r} \partial y^{r}} p\left(\xi^{\prime}, \eta^{\prime}\right)\right|\right\} .
$$

\section{Galerkin Method}

To derive Galerkin discretization based on interpolating scaling functions of one-dimensional HPDEs (1), the approximate solution can be written by projection operator $\mathscr{P}_{J}^{r}$, i.e.,

$$
w(x, t) \approx \mathscr{P}_{J}^{r}(w)(x, t)=\Phi_{J}^{r T}(x) W \Phi_{J}^{r}(t),
$$

where components of the square matrix $W$ of order $N$ are unknown coefficients that should be found and the superscript $T$ means transpose. Taking the second derivative with respect to $x$ and $t$ from both sides of equation (17), one can write

$$
\begin{gathered}
w_{t t}(x, t) \approx \mathscr{P}_{J}^{r}\left(w_{t t}\right)(x, t)=\Phi_{J}^{r T}(x) W D_{\phi}^{2} \Phi_{J}^{r}(t), \\
w_{x x}(x, t) \approx \mathscr{P}_{J}^{r}\left(w_{x x}\right)(x, t)=\Phi_{J}^{r T}(x) D_{\phi}^{T^{2}} W \Phi_{J}^{r}(t),
\end{gathered}
$$

where $D_{\phi}$ is the operational matrix of the derivative for interpolating scaling functions that are introduced in [17]. By substituting (18) in (1) and projecting the function $p$ to the space $V_{J}^{r}$ using projection $\mathscr{P}_{J}^{r}$, we obtain

$$
\Phi_{J}^{r T}(x) W D_{\phi}^{2} \Phi_{J}^{r}(t)-\Phi_{J}^{r T}(x) D_{\phi}^{T^{2}} W \Phi_{J}^{r}(t)=\Phi_{J}^{r T}(x) P \Phi_{J}^{r}(t) .
$$

Now, we introduce the residual function

$$
\begin{aligned}
r_{J}^{r}:= & \Phi_{J}^{r T}(x) W D_{\phi}^{2} \Phi_{J}^{r}(t)-\Phi_{J}^{r T}(x) D_{\phi}^{T^{2}} W \Phi_{J}^{r}(t) \\
& -\Phi_{J}^{r T}(x) P \Phi_{J}^{r}(t) .
\end{aligned}
$$

Note that $\mathscr{P}_{J}^{r}(v)=0\left(v \in V_{J}^{r}\right)$ if $\forall 1 \leq i \leq N\left\langle v,\left[\Phi_{J}^{r}\right]_{i}\right\rangle=0$. Multiplying (20) by $\Psi_{J}^{r T}(t)$ and $\Psi_{J}^{r T}(x)$ from the left and right, respectively, and integrating over $I_{J, b}, b \in \mathscr{B}_{J}$, we obtain a linear system as follows:

$$
\Lambda:=W D_{\phi}^{2}-D_{\phi}^{T^{2}} W-P=0,
$$

where we employ orthonormality of interpolating scaling functions and the local support of these bases. Equation (21) gives $N^{2}$ equations in which $4 N$ of these equations are linearly dependent. These equations are substituted using the conditions governing the problem. One can find $4 \mathrm{~N}$ extra equations utilizing the projection $\mathscr{P}_{J}^{r}$ for known functions $q, g, h$, and $k$ as follows:

$$
\begin{aligned}
w(x, 0) & =q(x), \longrightarrow \Phi_{J}^{r T}(x) W \Phi_{J}^{r}(0)=\Phi_{J}^{r}(x)^{T} Q \\
w_{t}(x, 0) & =g(x), \longrightarrow \Phi_{J}^{r T}(x) W D_{\phi} \Phi_{J}^{r}(0)=\Phi_{J}^{r}(x)^{T} G, \\
w(0, t) & =h(t), \longrightarrow \Phi_{J}^{r T}(0) W \Phi_{J}^{r}(t)=H^{T} \Phi_{J}^{r}(t), \\
\int_{0}^{1} w(x, t) & =k(x), \longrightarrow \Phi_{J}^{r T}(1) I_{\phi}^{T} W \Phi_{J}^{r}(t)=K^{T} \Phi_{J}^{r}(x),
\end{aligned}
$$

where $I_{\phi}$ is the operational matrix of the integral for interpolating scaling functions introduced in [14]. Because the interpolating scaling functions are linearly independent, we have, from (22), $4 N$ independent equations:

$$
\begin{aligned}
& \Gamma_{1}:=W \Phi_{J}^{r}(0)-Q=0, \Gamma_{2}:=W D_{\phi} \Phi_{J}^{r}(0)-G=0, \\
& \Gamma_{3}:=\Phi_{J}^{r T}(0) W-H^{T}=0, \Gamma_{4}:=\Phi_{J}^{r T}(1) I_{\phi}^{T} W-K^{T}=0 .
\end{aligned}
$$

Replacing $\Gamma_{1}$ and $\Gamma_{2}$ in the first and second columns of $\Lambda$ and $\Gamma_{3}$ and $\Gamma_{3}$ in the first and last rows of $\Lambda$, one can obtain the $N^{2}$ equations with $N^{2}$ unknowns. To find the unknowns and, equivalently, the approximate solution to the problem, this system must be solved by a convenient method.

Theorem 1. Let $f \in C^{r}(\Omega \times[0,1])$. Further suppose that $w(x, t)$, the solution of equation (1), is $r$-times derivative. The Galerkin method presented in this section applied to equation (1) is convergent.

Proof. To prove the Galerkin method convergence presented in this section, subtracting (1) from $\mathscr{P}_{J}^{r}\left(w_{t t}-w_{x x}-p\right)=0$, we obtain

$$
\begin{aligned}
\left|e_{J}^{r}\right| & =\left|w_{t t}-\mathscr{P}_{J}^{r}\left(w_{t t}\right)-w_{x x}-\mathscr{P}_{J}^{r}\left(w_{x x}\right)-p+\mathscr{P}_{J}^{r}(p)\right| \\
& =\left|e_{J t t}-e_{J x x}-p+\mathscr{P}_{J}^{r}(p)\right|,
\end{aligned}
$$

where $e_{J}:=w-\mathscr{P}_{J}^{r}(w)$. Using the triangle inequality and taking $L^{2}$-norm from both sides yield

$$
\left\|e_{J}^{r}\right\| \leq\left\|e_{J t t}+e_{J x x}\right\|+\left\|p-\mathscr{P}_{J}^{r}(p)\right\| .
$$

Now, suppose that

$$
e_{J}(x, t) \approx \mathscr{P}_{J}^{r}\left(e_{J}\right)(x, t)=\Phi_{J}^{r T}(x) E \Phi_{J}^{r}(t),
$$

where $E$ is the $(N \times N)$ matrix, and thus, one can write 
TABle 1: Comparison of the $L_{\infty}$ error for Example 1.

\begin{tabular}{lccccc}
\hline & \multicolumn{3}{c}{ Presented method } & \multicolumn{2}{c}{ Dehghan and Lakestani [1] } \\
& $J=2$ & $J=3$ & $J=4$ & $J=2$ & $J=3$ \\
\hline 0.2 & $8.6 e-4$ & $4.2 e-6$ & $1.6 e-7$ & $9.4 e-3$ & $1.9 e-4$ \\
0.4 & $1.0 e-3$ & $1.1 e-6$ & $3.4 e-8$ & $6.0 e-3$ & $9.3 e-5$ \\
0.6 & $8.4 e-4$ & $5.6 e-7$ & $4.2 e-8$ & $7.5 e-3$ & $9.3 e-5$ \\
0.8 & $4.3 e-4$ & $3.9 e-6$ & $1.0 e-7$ & $7.7 e-3$ & $1.9 e-4$ \\
1.0 & $3.2 e-4$ & $6.9 e-7$ & $5.9 e-8$ & $6.5 e-3$ & $8.10-5$ \\
\hline
\end{tabular}

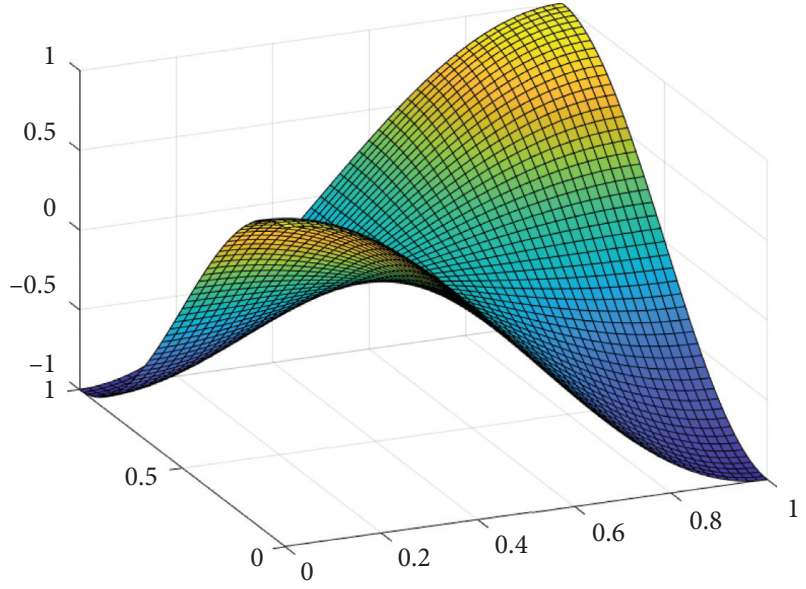

(a)

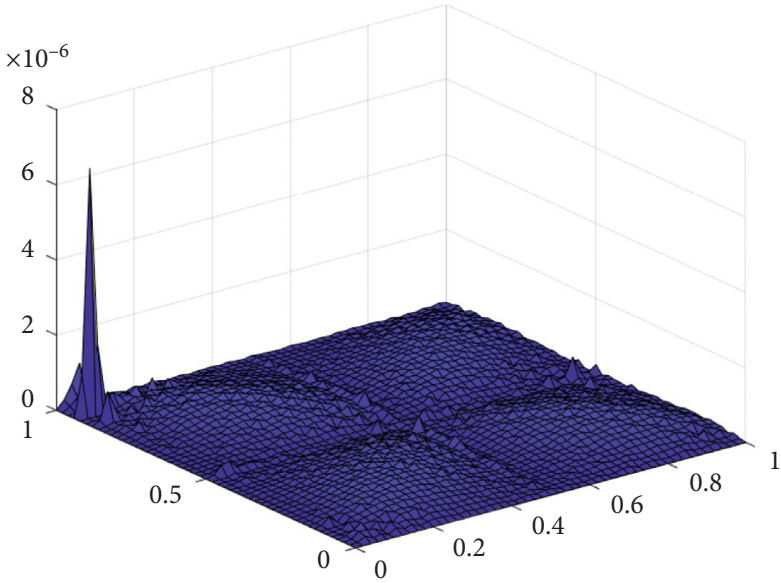

(b)

Figure 1: Plot of the approximate solution (a) and $L_{\infty}$ errors (b) taking $r=5$ and $J=4$ for Example 1.

$$
\begin{aligned}
\left\|e_{J}^{r}\right\| & \leq\left\|E D_{\phi}^{2}\right\|+\left\|D_{\phi}^{2^{T}} E\right\|+\left\|p-P_{J}^{r}(p)\right\| \\
& \leq 2\|E\|\left\|D_{\phi}^{2}\right\|+\left\|p-\mathscr{P}_{J}^{r}(p)\right\|,
\end{aligned}
$$

where we applied the orthonormality of bases. The operational matrix of the derivative for interpolating scaling functions is invertible [17], and its norm is finite. Let $\left\|D_{\phi}^{2}\right\|=\eta$; then, using (15), we can find

$$
\left\|e_{J}^{r}\right\| \leq \kappa \frac{2^{1-r J}}{4^{r} r !}\left(2+\frac{2^{1-J r}}{4^{r} r !}\right)
$$

where $\kappa$ is a constant.

\section{Numerical Results}

To illustrate the validity, efficiency, and accuracy of the proposed method, we give some numerical examples. The $L_{\infty}$ errors in the given tables and figures are

$$
e_{J}:=\left|w(x, t)-w_{J}^{r}(x, t)\right|,
$$

where $w_{J}^{r}(x, t)$ and $w(x, t)$ are the approximate and exact solutions at the point $(x, t)$, respectively.

Example 1. Let us consider hyperbolic partial differential equation (1) with

$$
\begin{cases}q(x):=\cos (\pi x), & g(x):=0, x \in \Omega, \\ h(t):=\cos (\pi t), & k(t):=0, t \in[0,1],\end{cases}
$$

and also $p(x, t):=0$. The exact solution of this problem is given in [1] as

$$
w(x, t):=\frac{1}{2}[\cos (\pi(x+t))+\cos (\pi(x-t))] .
$$

Table 1 shows the comparison of our results with those obtained by Dehghan and Lakestani [1]. The result confirms that the presented method is very convenient and effective. Taking $r=5$ and $J=4$, the approximate solution and $L_{\infty}$ error are shown in Figure 1. The effects of the multiplicity parameter $r$ and refinement level $J$ on the $L_{\infty}$ error are shown in Figure 2.

Example 2. Consider hyperbolic partial differential equation (1) with

$$
\begin{cases}q(x):=\sin (\pi x), & g(x):=-\frac{1}{2} \sin (\pi x), x \in \Omega, \\ h(t):=0, & k(t):=\frac{2}{\pi} e^{-t / 2}, t \in[0,1],\end{cases}
$$

and $p(x, t):=\left(1 / 4+\pi^{2}\right) e^{-t / 2} \sin (\pi x)$. The exact solution of this problem is given in [1] as

$$
w(x, t):=e^{-t / 2} \sin (\pi x) .
$$




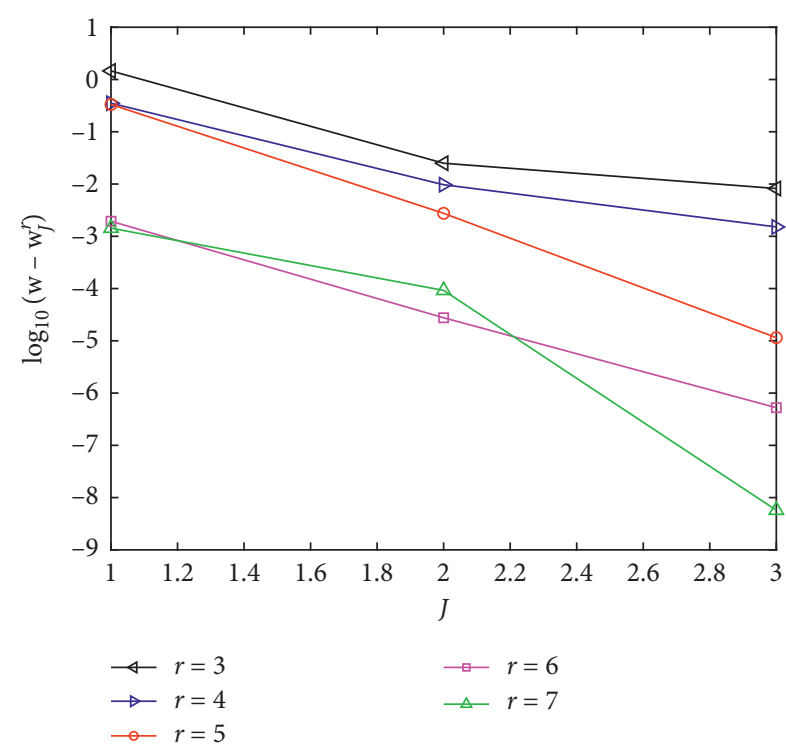

(a)

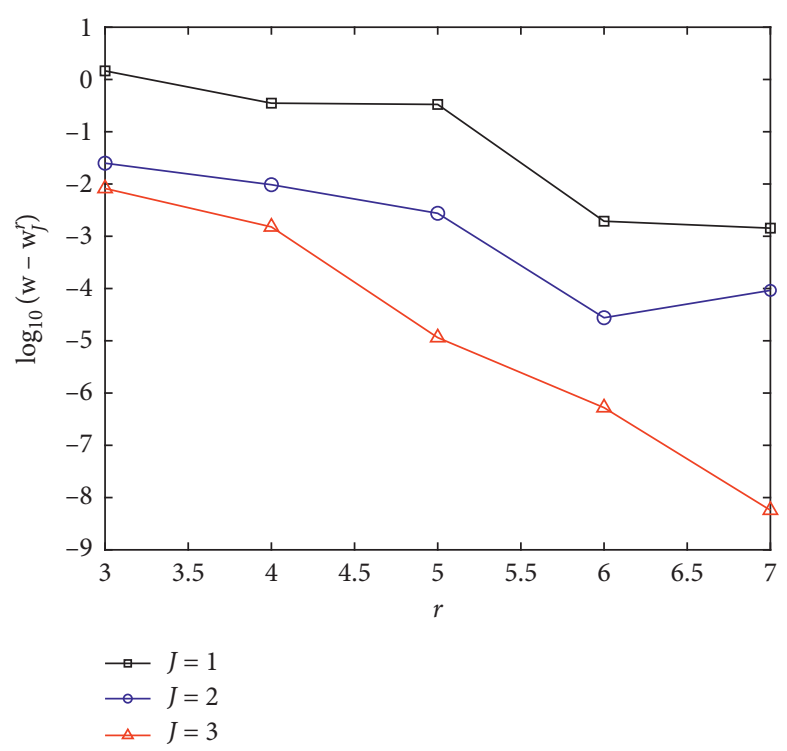

(b)

Figure 2: Effects of parameters $r$ and $J$ on the $L_{\infty}$ error for Example 1.

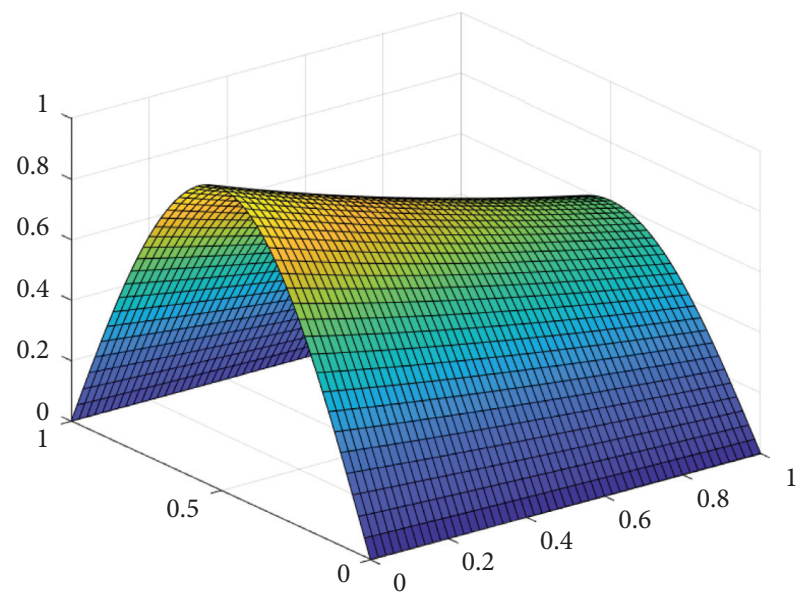

(a)



(b)

FIGURE 3: Plot of the approximate solution (a) and $L_{\infty}$ errors (b) taking $r=7$ and $J=3$ for Example 2.

The approximate solution of Example 1 has been plotted in Figure 3. In Table 2, results are compared with the cubic B-spline scaling function method [1] in terms of $L_{\infty}$ errors at different times. The effects of the refinement level $J$ and multiplicity parameter $r$ on $L_{\infty}$ errors are given in Figure 4 .

Example 3. To show the ability and accuracy of the proposed method, let us consider the following HPDEs [21]:

$$
w_{t t}(x, t)=w_{x x}(x, t),
$$

with initial conditions

$$
w(x, 0)=0, w_{t}(x, 0)=\pi \cos (\pi x), \quad x \in[0,1],
$$

boundary condition

$$
w(0, t)=\sin (\pi t)
$$

and also the integral condition

$$
\int_{0}^{1} w(x, t) \mathrm{d} x=0
$$

The exact solution is $\cos (\pi x) \sin (\pi t)$.

The absolute value of errors obtained by the presented method is compared with others in Table 3. Because interpolating scaling functions are flexible in choosing the multiplicity parameter $r$ and refinement level $J$, it is obvious that our method gives better results than others. In Table 4, we report the absolute value of errors for different values of $x$ at $t=1$ taking different values for $r$ and $J$. Finally, we illustrate the approximate solution and absolute error in Figure 5. 
TABle 2: Comparison of the $L_{\infty}$ error for Example 2.

\begin{tabular}{lccccc}
\hline & \multicolumn{3}{c}{ Presented method } & \multicolumn{2}{c}{ Dehghan and Lakestani [1] } \\
& $J=2$ & $J=3$ & $J=4$ & $J=2$ & $J=3$ \\
\hline 0.2 & $1.6 e-5$ & $7.8 e-8$ & $5.6 e-10$ & $1.9 e-3$ & $2.7 e-4$ \\
0.4 & $5.3 e-6$ & $9.5 e-8$ & $1.1 e-9$ & $5.3 e-3$ & $4.0 e-5$ \\
0.6 & $2.1 e-6$ & $7.6 e-9$ & $4.4 e-9$ & $5.0 e-3$ & $1.2 e-4$ \\
0.8 & $1.3 e-5$ & $1.1 e-7$ & $7.3 e-10$ & $2.4 e-3$ & $6.7 e-4$ \\
1.0 & $4.3 e-6$ & $9.3 e-9$ & $4.4 e-9$ & $1.5 e-2$ & $1.9 e-6$ \\
\hline
\end{tabular}

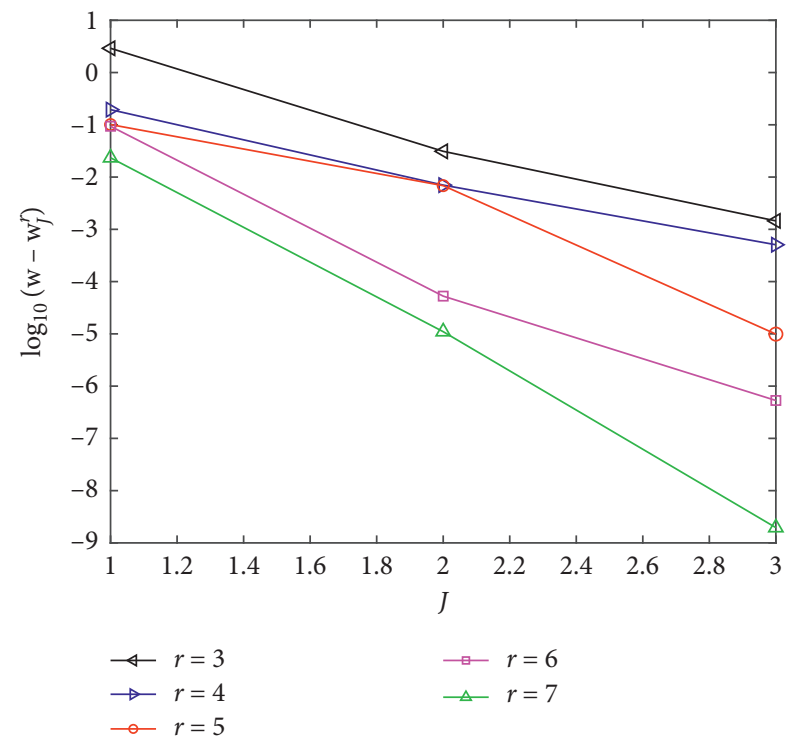

(a)

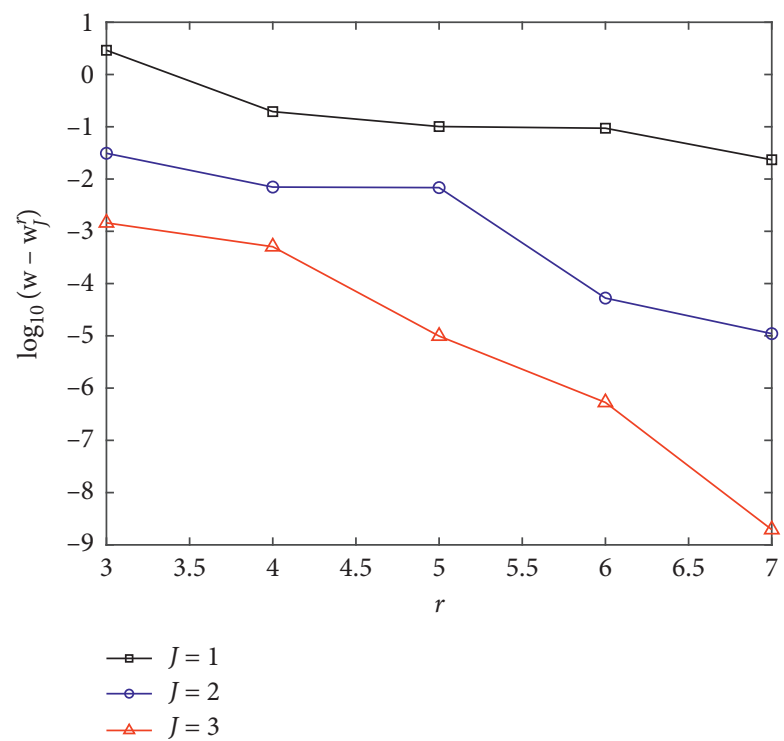

(b)

FIgURE 4: Effects of parameters $r$ and $J$ on the $L_{\infty}$ error for Example 2.

TABle 3: Comparison of the absolute value of errors at $t=1 / 2$ for Example 3.

\begin{tabular}{|c|c|c|c|}
\hline$x$ & $\begin{array}{l}\text { Presented method } \\
\qquad r=9\end{array}$ & $\begin{array}{l}\text { Turkyilmazoglu [21] } \\
\qquad r=9\end{array}$ & $\begin{array}{l}\text { Dehghan and Lakestani [1] } \\
\qquad r=12\end{array}$ \\
\hline 0.2 & $1.5 e-9$ & $6.4 e-8$ & $2.0 e-5$ \\
\hline 0.4 & $1.6 e-9$ & $2.1 e-8$ & $3.8 e-5$ \\
\hline 0.6 & $1.9 e-9$ & $2.1 e-8$ & $3.8 e-5$ \\
\hline 0.8 & $1.9 e-9$ & $6.4 e-8$ & $2.0 e-5$ \\
\hline
\end{tabular}

TABLE 4: The absolute value of errors at $t=1$ for Example 3.

\begin{tabular}{|c|c|c|c|c|c|c|}
\hline \multirow{2}{*}{$x$} & \multicolumn{3}{|c|}{$r=7$} & \multicolumn{3}{|c|}{$r=9$} \\
\hline & $J=1$ & $J=2$ & $J=3$ & $J=1$ & $J=2$ & $J=3$ \\
\hline 0.2 & $1.6 e-4$ & $1.2 e-6$ & $1.2 e-8$ & $4.4 e-6$ & $2.2 e-9$ & $2.5 e-9$ \\
\hline 0.4 & $4.4 e-5$ & $5.9 e-7$ & $4.3 e-9$ & $4.3 e-6$ & $9.0 e-10$ & $3.1 e-9$ \\
\hline 0.6 & $2.1 e-4$ & $5.9 e-7$ & $4.7 e-9$ & $5.3 e-6$ & $8.9 e-10$ & $4.5 e-9$ \\
\hline 0.8 & $7.4 e-5$ & $1.3 e-6$ & $1.2 e-8$ & $1.2 e-5$ & $2.4 e-9$ & $4.3 e-9$ \\
\hline 1.0 & $1.4 e-3$ & $5.4 e-7$ & $7.0 e-10$ & $5.0 e-5$ & $9.1 e-10$ & $1.1 e-10$ \\
\hline
\end{tabular}




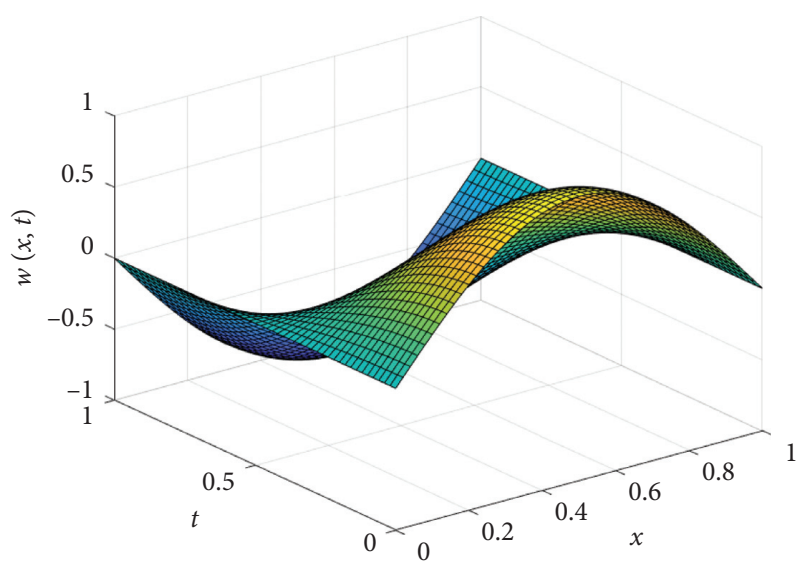

(a)

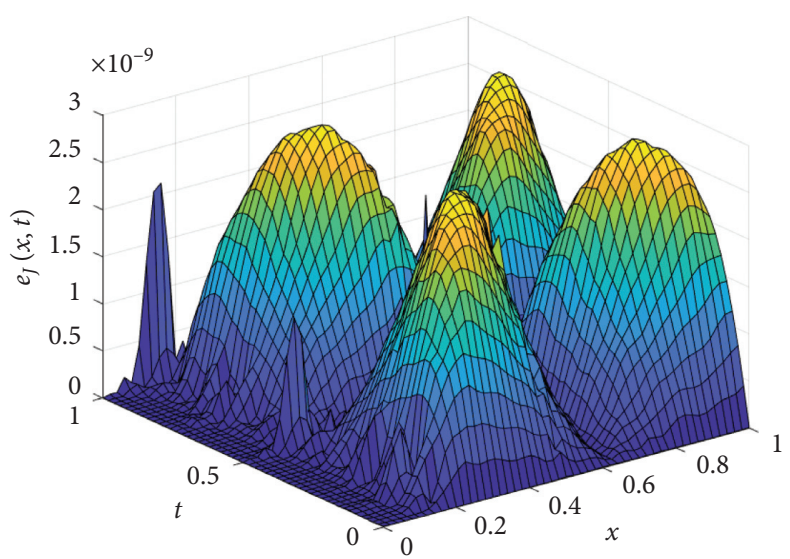

(b)

Figure 5: Plot of the approximate solution (a) and absolute value of errors (b) taking $r=9$ and $J=2$ for Example 3 .

\section{Conclusion}

Interpolating scaling functions are applied to obtain the approximate solution of one-dimensional hyperbolic partial differential equations with a nonlocal conservation condition. The convergence analysis is investigated, and the numerical results are compared with existing methods. It shows that the proposed method is much more flexible than others, and the results show that the proposed method has better accuracy than other methods.

\section{Data Availability}

No data were used to support this study.

\section{Conflicts of Interest}

The author declares no conflicts of interest.

\section{Acknowledgments}

This project was supported by the Researchers Supporting Project number (RSP-2020/210), King Saud University, Riyadh, Saudi Arabia.

\section{References}

[1] M. Dehghan and M. Lakestani, "The use of cubic B-spline scaling functions for solving the one-dimensional hyperbolic equation with a nonlocal conservation condition," Numerical Methods for Partial Differential Equations, vol. 23, no. 6, pp. 1277-1289, 2007.

[2] M. Lakestani, B. N. Saray and B. N. Saray, Numerical solution of telegraph equation using interpolating scaling functions," Computers and Mathematics with Applications, vol. 60, no. 7, pp. 1964-1972, 2010.

[3] W.-T. Ang, "A numerical method for the wave equation subject to a non-local conservation condition," Applied Numerical Mathematics, vol. 56, no. 8, pp. 1054-1060, 2006.

[4] M. Dehghan, "A computational study of the one-dimensional parabolic equation subject to nonclassical boundary specifications," Numerical Methods for Partial Differential Equations, vol. 22, no. 1, pp. 220-257, 2006.

[5] M. Dehghan, "On the solution of an initial-boundary value problem that combines Neumann and integral condition for the wave equation," Numerical Methods for Partial Differential Equations, vol. 21, no. 1, pp. 24-40, 2005.

[6] S. Mesloub and A. Bouziani, "Mixed problem with integral conditions for a certain class of hyperbolic equations," Journal of Applied Mathematics, vol. 1, no. 3, pp. 107-116, 2001.

[7] A. Ashyralyev and N. Aggez, "Finite difference method for hyperbolic equations with the nonlocal integral condition," Discrete Dynamics in Nature and Society, vol. 2011, Article ID 562385, 15 pages, 2011.

[8] A. H. Bhrawy, M. A. Alghamdi, and E. S. Alaidarous, "An efficient numerical approach for solving nonlinear coupled hyperbolic partial differential equations with nonlocal conditions," Abstract and Applied Analysis, vol. 2014, p. 14, Article ID 295936, 2014.

[9] E. H. Doha, R. M. Hafez, and Y. H. Youssri, "Shifted Jacobi spectral-Galerkin method for solving hyperbolic partial differential equations," Computers \& Mathematics with Applications, vol. 78, no. 3, pp. 889-904, 2019.

[10] S. Singh, V. K. Patel, and V. K. Singh, "Application of wavelet collocation method for hyperbolic partial differential equations via matrices," Applied Mathematics and Computation, vol. 320, no. 1, pp. 407-424, 2018.

[11] M. Turkyilmazoglu, "Parabolic partial differential equations with nonlocal initial and boundary values," International Journal of Computational Methods, vol. 12, no. 5, Article ID 1550024, 2015.

[12] B. N. Saray, "An efficient algorithm for solving Volterra integro-differential equations based on Alpert's multi-wavelets Galerkin method," Journal of Computational and Applied Mathematics, vol. 348, pp. 453-465, 2019.

[13] B. N. Saray, "Sparse multiscale representation of Galerkin method for solving linear-mixed Volterra-Fredholm integral equations," Mathematical Methods in the Applied Sciences, vol. 43, no. 5, pp. 2601-2614, 2020.

[14] B. N. Saray, M. Lakestani, and M. Razzaghi, "Sparse representation of system of Fredholm integro-differential equations by using alpert multiwavelets," Computational Mathematics and Mathematical Physics, vol. 55, no. 9, pp. 1468-1483, 2015. 
[15] B. N. Saray and J. Manafian, "Sparse representation of delay differential equation of pantograph type using multiwavelets Galerkin method," Engineering Computation, vol. 35, no. 2, pp. 887-903, 2018.

[16] B. Alpert, G. Beylkin, R. Coifman, and V. Rokhlin, "Waveletlike bases for the fast solution of second-kind integral equations," SIAM Journal on Scientific Computing, vol. 14, no. 1, pp. 159-184, 1993.

[17] B. Alpert, G. Beylkin, D. Gines, and L. Vozovoi, "Adaptive solution of partial differential equations in multiwavelet bases," Journal of Computational Physics, vol. 182, no. 1, pp. 149-190, 2002.

[18] N. Hovhaüller and R. Schäfer, "Aaptive multiresolution discontinuous Galerkin schemes for conservation laws," Mathematics of Computation, vol. 83, no. 285, pp. 113-151, 2014.

[19] S. H. Seyedi, B. Nemati Saray, and A. Ramazani, "High-accuracy multiscale simulation of three-dimensional squeezing carbon nanotube-based flow inside a rotating stretching channel," Mathematical Problems in Engineering, vol. 2019, Article ID 9890626, 18 pages, 2019.

[20] S. H. Seyedi, B. Nemati Saray, and A. Ramazani, "On the multiscale simulation of squeezing nanofluid flow by a highprecision scheme," Powder Technology, vol. 340, pp. 264-273, 2018.

[21] M. Turkyilmazoglu, "Hyperbolic partial differential equations with nonlocal mixed boundary values and their analytic approximate solutions," International Journal of Computational Methods, vol. 15, no. 1, Article ID 1850003, 2018. 